\title{
Lithium wall conditioning by high frequency pellet injection in RFX-mod
}

\author{
P. Innocente ${ }^{a^{*}}$, D.K. Mansfield ${ }^{b}$, A.L. Roquemore ${ }^{b}$, M. Agostini ${ }^{a}$, S. Barison ${ }^{c}$, A. Canton ${ }^{a}$, L. $^{2}$ \\ Carraro $^{a}$, R. Cavazzana, G. De Masi ${ }^{\mathrm{a}}$, A. Fassina ${ }^{\mathrm{a}}$, S. Fiameni ${ }^{\mathrm{c}}$, L. Grando ${ }^{\mathrm{a}}$, B. Rais ${ }^{\mathrm{a}}$, F. \\ Rossetto $^{\mathrm{a}}$, P. Scarin ${ }^{\mathrm{a}}$ \\ ${ }^{a}$ Consorzio RFX, Corso Stati Uniti 4, 35127 Padova, Italy \\ ${ }^{b}$ Princeton Plasma Physics Laboratory, Princeton, New Jersey 08543, USA \\ ${ }^{c}$ CNR-IENI, Corso Stati Uniti 4, 35127 Padova, Italy
}

\begin{abstract} further optimizations are required.

PACS: 52.55.Lf, 52.25.Fi

PSI-20 keywords: RFX, Lithium, Pellet, Density control, Wall conditioning

*Corresponding author address: Corso Stati Uniti, 5, 35127, Padova, Italy

*Corresponding author E-mail: paolo.innocente@igi.cnr.it

Presenting author: Paolo Innocente

Presenting author e-mail: paolo.innocente@igi.cnr.it
\end{abstract}

In the RFX-mod reversed field pinch experiment, lithium wall conditioning has been tested with multiple scopes: to improve density control, to reduce impurities and to increase energy and particle confinement time. Large single lithium pellet injection, lithium capillary-pore system and lithium evaporation has been used for lithiumization. The last two methods, which presently provide the best results in tokamak devices, have limited applicability in the RFX-mod device due to the magnetic field characteristics and geometrical constraints. On the other side, the first mentioned technique did not allow injecting large amount of lithium. To improve the deposition, recently in RFX-mod small lithium multi-pellets injection has been tested. In this paper we compare lithium multi-pellets injection to the other techniques. Multi-pellets gave more uniform Li deposition than evaporator, but provided similar effects on plasma parameters, showing that 


\section{Introduction}

Lithium is a first wall material that can improve plasma performance in magnetically confined devices. The first positive results were obtained in TFTR [1] by injecting few lithium pellets in conditioning discharges. Since then many different techniques have been developed to improve the lithium deposition. Lithium evaporation has been introduced to increase the amount of lithium deposited over the structural wall/divertor materials [2], then lithium capillary-pore systems (LCPS) [3,4] and free liquid lithium surfaces (LLS) [5] have been used to continuously refurbish with 'fresh' lithium the wall. In RFX-mod some of these techniques have been experimented following a similar path. After the first positive results obtained by injecting relatively large right-circular cylinders $(\varnothing=1.5 \mathrm{~mm}, \mathrm{~L}=5 \mathrm{~mm})$ lithium pellets by a single pellet injector [6], lithiumization moved to LCPS and evaporator [7]. However pellet injection has been recently reconsidered because the magnetic and geometrical characteristics of the RFX-mod reversed field pinch make it difficult to obtain a uniform lithium wall coating by other means. The RFX-mod experiment [8] is a circular cross section Reversed Field Pinch (RFP) device with major/minor radius 2.0/0.46 m, maximum plasma current of $2 \mathrm{MA}$ and a first wall entirely covered by graphite tiles. Owing to the high aspect ratio of the device, a high number of evaporators would be required to provide a uniform lithium deposition; furthermore in RFX-mod it is very difficult to install shutters on vacuum vessel windows to prevent windows darkening during evaporation. For this reason lithiumization with this method is possible over a small fraction of the wall only. A different restriction applies to the LCPS and LLS methods, due to the fact that the magnetic field at the edge of a RFP is poloidal: lithium deposition is toroidally limited to few degrees away from LCPS/LLS. Oppositely, single lithium pellet injection proved to be able to realize a uniform lithium coating over the whole wall surface. The drawback is that conventional injectors require a high number of conditioning discharges to reach an adequately 
thick lithium coating. Recently, to overcome this problem, in RFX-mod the new multi-pellet injector (MPI) developed by the Princeton Plasma Physics Laboratory originally for ELM pacing [9] has been tested. The aims of this technique were: 1) the injection of an increased amount (at least a few grams) of lithium deep into the plasma core of helium conditioning discharges to provide a uniform thick lithium deposition on the wall; 2) lithium injection also in hydrogen discharges to continuously refurbish the wall with 'fresh' lithium during normal operation.

In this paper we first present the experimental setup of the multi-pellet injector in RFX-mod and the injection characteristics, then we analyze the results in terms of lithium deposition on the wall and its effects on the plasma.

\section{Experimental setup}

A detailed description of the new multi-pellet injector developed by the Princeton Plasma Physics Laboratory can be found in Ref. 9, where its application on EAST tokamak device for ELM pacing is described. In that case $0.7 \mathrm{~mm}$ diameter lithium spheres were injected at a frequency of $25 \mathrm{~Hz}$. These parameters are inadequate to inject a large amount of lithium in the RFX-mod discharges, which have typical duration of 0.3-0.5 s. To increase the injection rate, lithium spheres of $1.0 \mathrm{~mm}$ diameter (the maximum one commercially available for the present) have been selected; furthermore the dropper frequency has been increased by installing a piezoelectric disc (PZD) with a $4.0 \mathrm{~mm}$ central aperture instead of the $2.5 \mathrm{~mm}$ one used on EAST. Initially a serious problem was the high number of pellets lost in the vacuum duct between the impeller and the plasma, due to the spread in the granule injection angle and the small size $(38 \mathrm{~mm})$ of the CF40 vacuum port available for the installation. To maximize the injection efficiency, the RFXmod interface has been modified in order to use the whole aperture of the vacuum port and the injector has been moved as close as possible to the vacuum vessel. With this arrangement, $1 \mathrm{~mm}$ lithium spheres have been injected in RFX-mod at the maximum frequency of $250 \mathrm{~Hz}$ for a 
lithium mass injection of $70 \mathrm{mg} / \mathrm{s}$. In each discharge lithium injection started at $-0.02 \mathrm{~s}$ and continued for all the discharge, typically lasting $0.35 \mathrm{~s}$, providing a deposition on the wall of about $25 \mathrm{mg} / \mathrm{discharge.} \mathrm{Compared} \mathrm{to} \mathrm{the} 4 \mathrm{mg} / \mathrm{discharge}$ previously obtained by means of the single pellet injector, this corresponds to a 7 times increase in deposition rate.

In RFX-mod one single lithiumization campaign using the MPI has been executed. At the beginning of the campaign lithium pellets have been injected in about 40 medium current $(\mathrm{I}=0.8$ 1.0 MA) helium discharges for total of $1.1 \mathrm{~g}$ of deposited lithium; then some high current (I=1.21.5 MA) hydrogen discharges have been executed to study the effect of lithium coating on plasma performance. The campaign continued with a succession of helium conditioning discharges and hydrogen performance ones. Lithium pellets were launched in most of the hydrogen discharges and in all the helium discharges executed in between, so that by the end of campaign a total amount of about $2.3 \mathrm{~g}$ of lithium was injected.

Helium discharges were optimized to maximize pulse duration at a value of plasma current low enough to allow good pellet penetration during at least a fraction of the discharges. In figure 1a a camera image of Li I emission shows the ablation of three pellets in a $800 \mathrm{kA}$ plasma current discharge (figure 1b). The camera image shows that pellet penetration is limited to the external plasma region, in agreement with numerical evaluation of pellet ablation shown in figure 2 , where the ablation rate (fig. 2a) is computed using the Parks et al's model [10] at five times during the discharge in fig. 1 . Figure 2 shows that lithium pellets can reach the plasma center at the end of the discharge only. This is different from the single pellet injection case [6] where pellets always reached the plasma center due to their bigger size. We will show later that this penetration difference slightly affects the toroidal uniformity of lithium deposition. An interesting feature of the small lithium MPI is that discharges are not significantly affected in terms of plasma parameters (see plasma current, temperature and density in fig. 1b-1d with and without 
pellet injection); this would allow the injection of lithium at a higher rate, and this is presently considered as a way to increase the thickness and the quality of the lithium coating.

To analyze the lithiumization performed by MPI and to compare it to the other techniques in terms of deposition thickness and toroidal uniformity, graphite samples have been inserted at two toroidal positions and exposed to discharges with lithium injection. The absolute lithium abundance on the surface of the samples has been measured by XPS (X-ray photoelectron spectroscopy) and lithium depth profiles have been measured by SIMS (Secondary Ions Mass Spectrometry). These measurements that give information integrated over many discharges were supplemented by time resolved LiI and $\mathrm{H}_{\alpha}$ measurements available for each discharge at three toroidal positions.

\section{Results and discussion}

Figure 3 shows the SIMS depth profile analysis of a graphite sample installed at $30^{\circ}$ in the toroidal direction from MPI and exposed to the lithiumization helium discharges. The SIMS is not calibrated, hence the traces cannot be used for an quantitative estimation of the lithium content. The lithium profile shows a peak at about $50 \mathrm{~nm}$ from the surface which would correspond to the theoretical thickness of a pure lithium layer considering the RFX-mod wall area and the amount of injected lithium. On the other hand the figure shows that the lithium is not deposited as a pure film, but it is mixed with carbon. The $\mathrm{Li} / \mathrm{C}$ ratio was estimated by rescaling the whole lithium profile to the total amount of injected lithium and assuming uniform toroidal and poloidal deposition, and it is indicated on the right y-axis of the same figure. The estimated ratio of $\mathrm{Li} / \mathrm{C}=7 \%$ on surface is in good agreement with $6.5 \%$ measured by XPS. The agreement between the XPS measurement and the rough estimation from SIMS profile and injected lithium confirms that the injected lithium was unable to completely cover the graphite but that $\mathrm{Li}$ 
intercalated into the graphite lattice. Presently it is unknown whether Li intercalates into graphite during deposition or if, as shown by Itou et al [11], it diffuses into the graphite after each discharge. It is nevertheless interesting to note that lithium influxes in discharges after lithiumization do not decrease with time since lithiumization (i.e. the discharge without Li injection executed 20", one night or three days in weekends after the conditioning one shows similar lithium influxes); this seems to indicate that lithium diffusion in the RFX-mod polycrystalline graphite is negligible or it happens in few minutes after the discharges (as it was also observed by Itou et al. but in highly oriented pyrolytic graphite).

Lithium depth profile on a sample exposed at $150^{\circ}$ in the toroidal direction from the injection section is similar to the previous one in shape, but with about half of the peak height. The same behavior of the profiles was obtained with the single pellet injector while the measured profiles were very different in the evaporator case. In the latter case a higher amount of $15.5 \mathrm{~g}$ of lithium was evaporated and a graphite sample at the same toroidal position of the evaporator showed a nearly pure lithium layer of a thickness of about $500 \mathrm{~nm}$ and a nearly zero carbon at the surface. But just $60^{\circ}$ toroidally away from the evaporator the lithium layer became thinner (about $100 \mathrm{~nm}$ ) and mixed with carbon. This means that by the evaporator it was possible to cover the graphite wall by lithium in a small part of the toroidal vessel only.

Li influx measurements for the three lithiumization methods, multiple-pellet injection, single pellet injection and evaporator, show correspondingly a small, negligible or high toroidal asymmetry in qualitative agreement with lithium deposition measured on samples by SIMS. In particular in the MPI case Li influxes measured $150^{\circ}$ far from injection section are about $1 / 6$ of those measured $30^{\circ}$ away (Fig. 4a). This confirms a small toroidal asymmetry in deposition that comes from the low penetration of some of the injected pellets. Differently, at the injection section and $180^{\circ}$ away $\mathrm{Li}$ influxes are very similar in the single-pellet case while in the 
evaporator case lithium influx is negligible $180^{\circ}$ away from the evaporator (Fig. 4b). These are again in agreement with SIMS measurements of a toroidal asymmetry which is negligible for the single pellet case and important for the evaporator case. Lithium influxes also show that $\mathrm{H}$ or $\mathrm{He}$ discharges are ineffective in re-distributing lithium in the toroidal direction: hundreds of discharges far from lithium evaporation Li influx is strongly reduced but its toroidal asymmetry is nearly the same as immediately after Li evaporation.

Due to the limited number of hydrogen discharges performed after MPI lithiumization and the relatively high fluctuation in discharge parameters (in particular in terms of electron density) typical of RFX-mod, it is not possible to obtain a statistically meaningful comparison of the performance with respect to the other lithiumization and/or wall conditioning methods. Nonetheless some indication can be extracted on the basis of single or of small group of discharges, confirming most of the observations on plasma parameters already found with the other lithiumization techniques. The lithium coated wall by MPI allowed better density control than carbon wall: figure 5 shows two consecutive hydrogen discharges with the same set up, in particular in the two discharges it was used the same amount of hydrogen gas and lithium injection. A lower plasma density resulted in the second discharge (figure 5b), a behavior never observed with non lithiumized carbon wall, where at constant hydrogen gas the plasma density increases nearly steadily discharge after discharge due to the progressive hydrogen accumulation in the graphite. The decrease in density observed in Li injection case shows that freshly injected and accumulated lithium are able to more than counter balance hydrogen accumulation proving an increased hydrogen trapping efficiency and a consequent reduced recycling.

Lithiumization performed by MPI provides an impurity reduction similar to that previously observed with the other lithiumization techniques and boronization. As an example figure 6 shows oxygen and carbon influxes measured for discharges performed far from a boronization $(\mathrm{C}$ 
wall), just after a wall boronization (B wall) and after MPI lithiumization (Li wall). On average lithium coating provides the lowest impurity influx with significant improvement with respect to the carbon case. This result agrees in particular with that of the past single pellet lithiumization, confirming the good toroidal uniformity of lithium deposition obtained with the pellet-based techniques.

Less clear effects, though comparable to those observed with the other lithiumization techniques, have been seen in terms of particle and energy confinement: some improvement has been seen in terms of particle confinement times but the error bars due to discharges variation are very large.

\section{Conclusions}

The high frequency Princeton Plasma Physics Laboratory multi-pellet injector has been tested in RFX-mod to set-up a lithiumization method able to realize a lithium thick $(50-100 \mathrm{~nm} / 1-2 \mathrm{gr})$ coating on the wall, toroidally and poloidally uniform, in one operation day of plasma conditioning discharges (about 20 discharges/day). The first experiments have shown a toroidal uniformity of lithium coating thickness nearly as good as that previously obtained with single large pellet injection, despite the smaller pellet size and, accordingly, the shorter pellet penetration. Toroidal uniformity was better that that obtained in lithium evaporation experiments, which showed a very high toroidal non-uniformity. In terms of injection rate, a seven times increase has been obtained compared to the single pellet injector but the rate is still insufficient to reach the target of more than $1 \mathrm{gr} /$ day. Measurements of lithium content on the surface and in depth of graphite samples exposed during lithiumization have shown that a mixed carbon/lithium layer has been obtained, as in the case of the single pellet lithium injection. Compared to the evaporator case, where a thick lithium layer close to the evaporation section and a mixed lithium/carbon layer far from it was realized, the MPI results indicate that the injected lithium was completely intercalated in the graphite lattice and that a higher amount of lithium would be 
necessary to completely cover the graphite wall. Experiments performed after lithium wall conditioning confirmed the improvements of plasma parameter in terms of lower impurity content and better density control. Yet the lack of a significant improvement in particle and confinement time can be ascribed to the lithium mixing into the graphite. For this reason a longer lithium injection campaign is foreseen to assess the advantages of lithium wall conditioning. An increase in lithium injection rate is also planned by injecting bigger lithium spheres custom made using a Lithium Pellet Production device [12]. Lithium spheres with a $1.5 \mathrm{~mm}$ diameter should double the mass injection rate and will provide deeper penetrating pellets, allowing injection in higher plasma current discharges which are longer in RFX-mod. In such a way a further increase of $\mathrm{Li}$ injection rate is expected.

\section{Acknowledgments}

This work was sponsored in part by the US Department of Energy and Princeton Plasma Physics Laboratory under Loan Number P12-03.

The authors wish to thank Dr. Charles H. Skinner of Princeton Plasma Physics Laboratory and Dr. Bruce Koel of Princeton University for their collaboration in surface analysis.

\section{References}

[1] D.K. Mansfield et al., Physics of Plasmas 3 (1996) 1892

[2] M.G. Bell et al., Plasma Physics Control. Fusion 51 (2009) 124054

[3] S.V. Mirnov et al., Plasma Phys. Control. Fusion 49 (2006) 821

[4] G. Mazzitelli et al., Nuclear Fusion 51 (2011) 073006

[5] Z. Sun et al., Journal of Nuclear Materials 438 (2013) S899

[6] S. Munaretto et al., Nuclear Fusion 52 (2012) 02301

[7] M.E. Puiatti, et al., Nuclear Fusion 53 (2013) 073001

[8] P. Sonato et al, Fusion Engineering and Design 161 (2003) 66

[9] D.K. Mansfield et al., Nuclear Fusion 53 (2013) 113023

[10] P.B. Parks, J.S. Leffler and R.K. Fisher, Nuclear Fusion 28 (1988) 477 
[11] N. Itou, H. Toyoda, K. Morita and H. Sugai, Journal Nuclear Material 290-293 (2001) 281

[12] P. Fiflis et al, Review Scientific Instruments 84 (2013) 063506 
Figure 1. Multi-pellet lithium injection: a) camera image showing the ablation of three lithium pellets in discharge \#34260. Main plasma parameter in the same discharge and in discharge \#34297 without pellet injection: b) plasma current; c) electron temperature; d) electron density. The shaded region shows the integration time of camera image.

Figure 2. Lithium pellet ablation computation at five times of discharge \#34260: a) Ablation rate versus plasma radius; b) Pellet radius.

Figure 3. SIMS depth profile measurement of lithium and carbon on a sample exposed to lithiumization helium discharges. On the left side ordinate SIMS measured Li and C counts are normalized to $\mathrm{O}$ counts, on the right side ordinate $\mathrm{Li} / \mathrm{C}$ concentration has been estimated from amount of injected lithium and integrated depth profile.

Figure 4. Lithium influxes at three toroidal positions from MPI and evaporator sections: a) He discharge, without Li injection, after 1 gr of a totally injected lithium; b) H discharge after $13.5 \mathrm{gr}$ of a totally evaporated lithium.

Figure 5. a) Plasma current and b) electron density of two following discharges executed with the same filling hydrogen gas and injecting Li.

Figure 6. a) Carbon and b) Oxygen influxes averaged for three set of discharges: executed far from boronization (circles), after boronization (diamonds), after lithiumization (squares). 
Figures

Figure 1

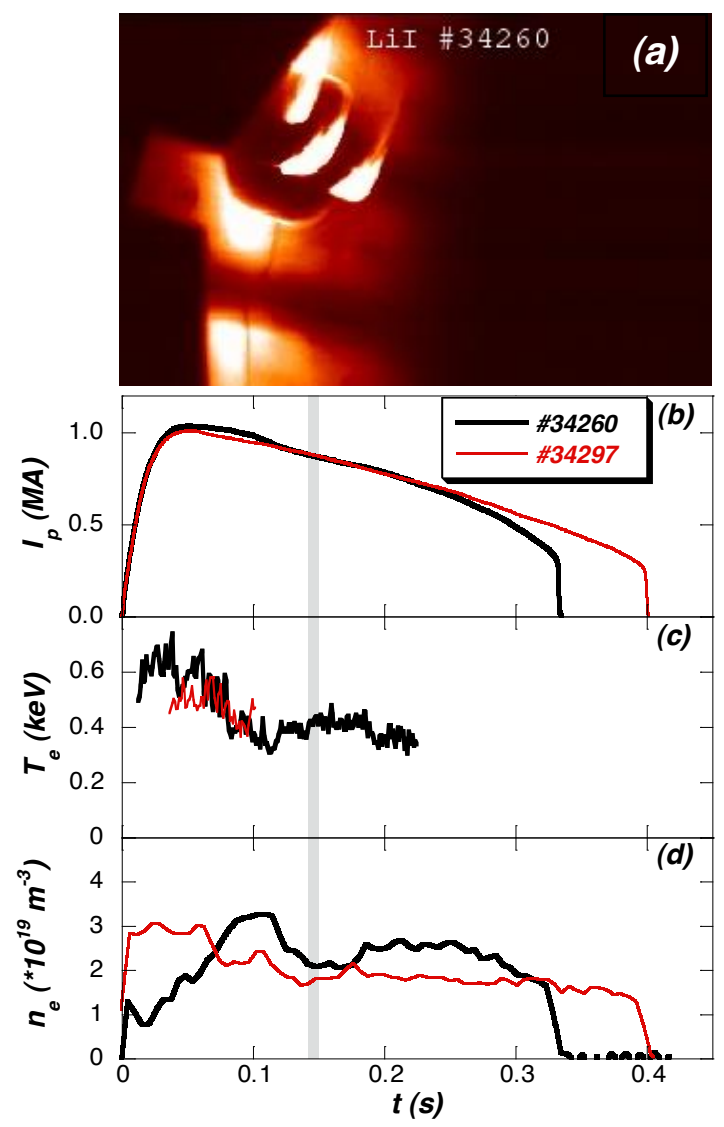


Figure 2

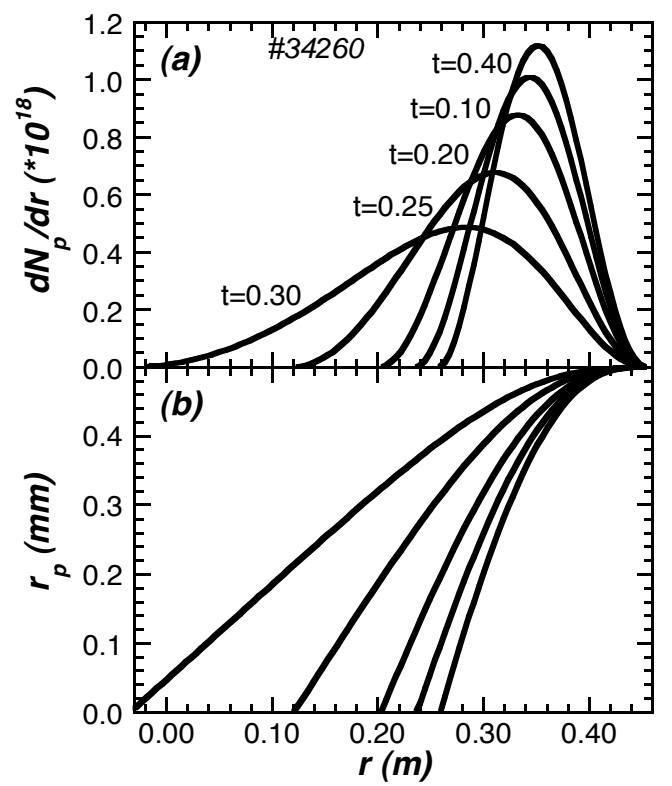


Figure 3

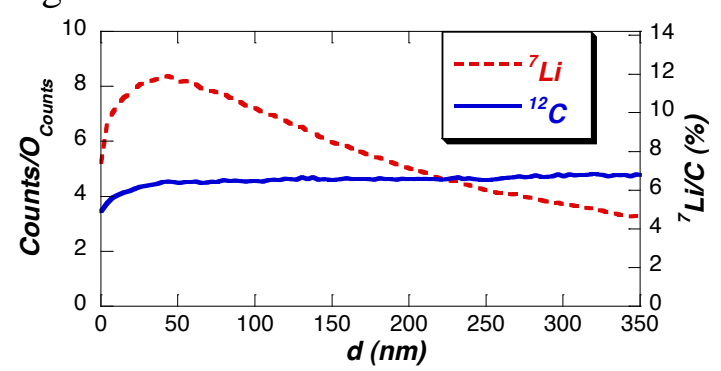


Figure 4

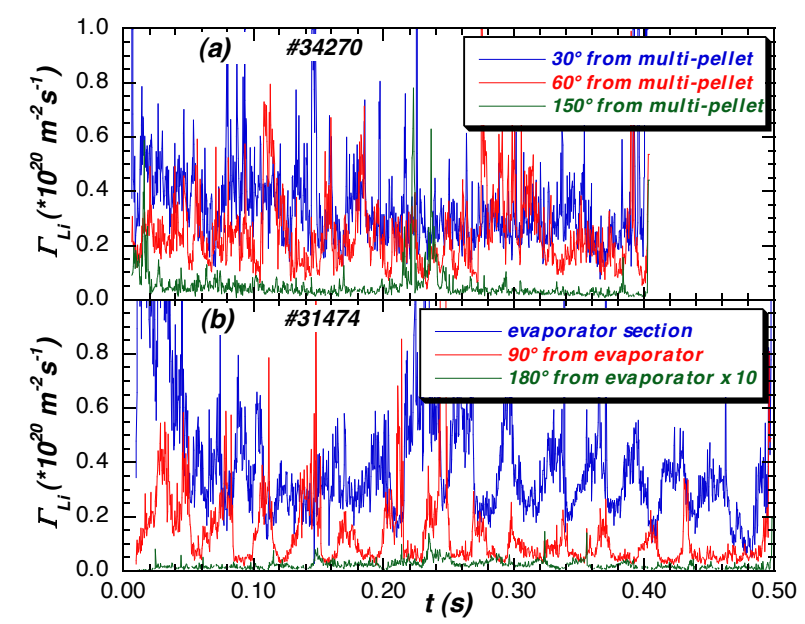


Figure 5

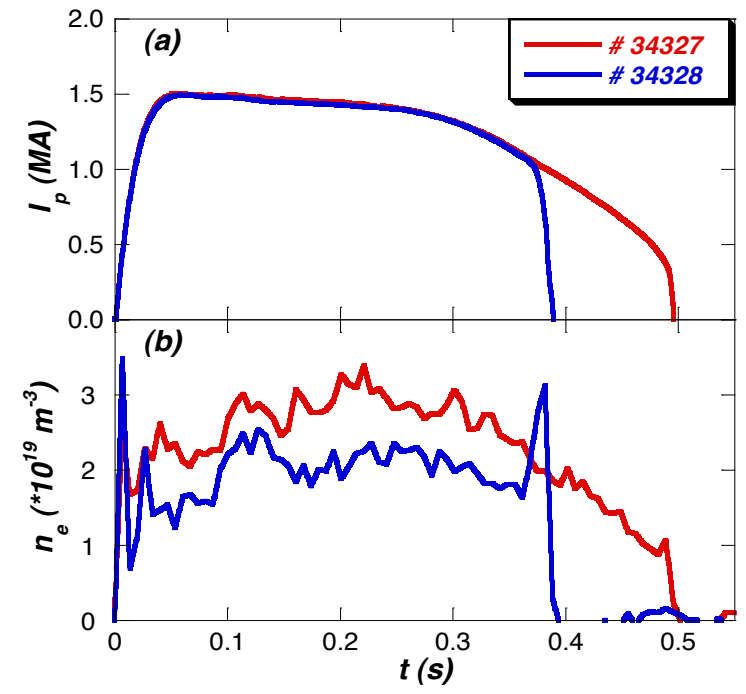


Figure 6

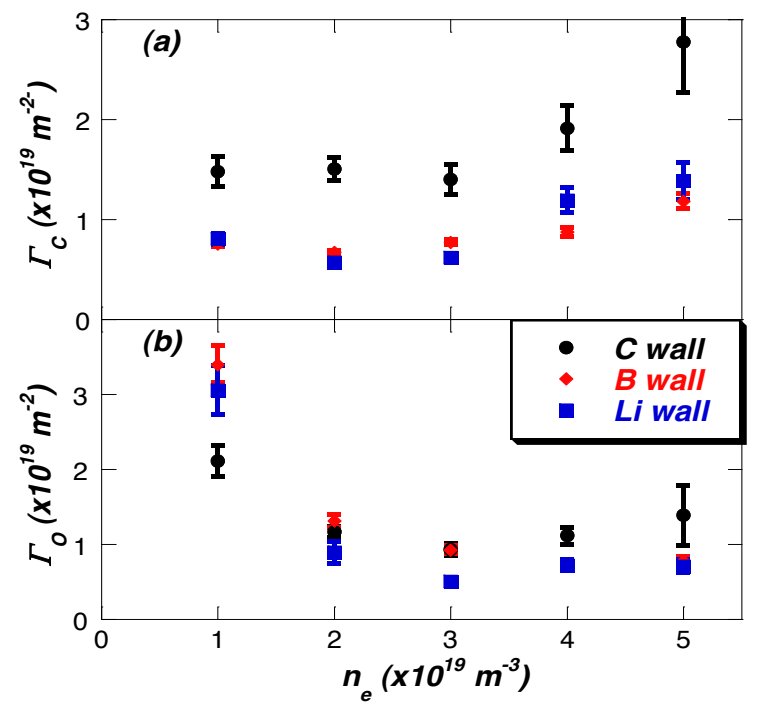

